\title{
Computational inference of regulator activity in a single input motif from gene expression data
} Raya Khanin*1, Veronica Vinciotti ${ }^{2}$, Vassilis Mersinias ${ }^{3}$, Colin Smith ${ }^{3}$ and Ernst Wit ${ }^{1}$

\author{
Address: ${ }^{1}$ Department of Statistics, 15 University Gardens, University of Glasgow, Glasgow G12 8QQ, UK., ${ }^{2}$ School of Information Systems, \\ Computing and Mathematics, Brunel University, Uxbridge, UB8 3PH, UK. and ${ }^{3}$ School of Biomedical and Molecular Sciences, University of Surrey, \\ Guildford, GU2 7XH, UK. \\ Email: Raya Khanin* - raya@stats.gla.ac.uk \\ * Corresponding author
}

from BioSysBio: Bioinformatics and Systems Biology Conference

Edinburgh, UK, 14-15 July 2005

Published: 21 September 2005

BMC Bioinformatics 2005, 6(Suppl 3):S7

This work deals with the computational modelling of gene regulation by transcription factor (regulator) in a single input motif (one regulator-many targets). The basic underlying problem is that the expression of a gene encoding the regulator provides only limited information about the activity of the regulator, which can be modified post-transcriptionally, for example, by phosphorylation. In the absence of reliable technology, it is of great importance to understand whether regulator activity can be inferred from gene expression data. We here develop a computational approach to reconstruct the regulator activity from gene expression data of the target genes in the SIM. We put forward a statistical framework that embeds the quantitative Michaelis-Menten model of the kinetics of gene regulation. The model uses the gene expression data on its original (rather than log) scale, employing the assumption of a log-normal noise distribution. For each target gene the maximum likelihood kinetic parameters of its regulation can be inferred independently given the profile of their common regulator. For a set of regulated target genes in the SIM, the regulator profile is reconstructed by maximizing the likelihood of the whole SIM.

The model is applied to two time series of the Streptomyces coelicolor bacterium: in one of them the transcriptional activator, cdaR, that is partly responsible for the production of one type of antibiotic, is knocked out. Our approach can be extended to include more complex regulatory relationships: multiple regulatory factors, competition, cooperativity. 